\title{
Rare Surgical Complication of In Vitro Fertilisation Treatment: Bladder Injury During Oocyte Retrieval
}

\author{
Tat Xin Ee ${ }^{a}$, Wuen Lynn Toh ${ }^{\text {b, d }}$, Jerry K.Y. Chan ${ }^{\text {a, c }}$
}

\begin{abstract}
Current literature suggests that surgical complications from oocyte retrievals (ORs) are uncommon. Here, we present a rare case of bladder injury during OR and its subsequent management. A 37-year-old nulliparous woman underwent assisted reproductive therapy (ART) for primary infertility secondary to anovulatory cycle. During OR, there was an inadvertent puncture of the bladder, with active intrabladder bleeding seen on transvaginal ultrasound. Bladder washout followed by continuous drainage was instituted with antibiotic coverage over several days. Bladder integrity ascertained through computer tomography urogram and cystoscopy were unremarkable. She was discharged well and continued with her fertility treatment. Transvaginal OR is associated with few complications. Bladder injury, albeit rare, can present with massive hematuria and hemodynamic instability. Early identification of the injury is key to management with insertion of the indwelling urinary catheter, hemodynamic resuscitation and bladder irrigation. With a urological multi-disciplinary approach, most bladder injuries can be resolved non-invasively.
\end{abstract}

Keywords: Reproductive medicine; Assisted reproductive therapy; In vitro fertilization; Oocyte retrieval; Gynaecology; Infertility; Bladder injury; Urology

\section{Introduction}

The International Committee for Monitoring Assisted Reproductive Technology (ICMART) estimates that 0.5 million children were born in the world from in vitro fertilization (IVF) procedures, and 2 million ART cycles were performed glob-

Manuscript submitted December 6, 2020, accepted December 22, 2020

Published online January 19, 2021

aDepartment of Reproductive Medicine, KK Women's and Children's Hospital, Singapore, Singapore

bDepartment of Obstetrics and Gynecology, KK Women's and Children's Hospital, Singapore, Singapore

${ }^{\mathrm{c} C a n c e r}$ and Stem Cell Biology, Duke-NUS Medical School, Singapore, Singapore

${ }^{\mathrm{d}}$ Corresponding Author: Wuen Lynn Toh, Department of Obstetrics and Gynecology, KK Women's and Children's Hospital, 100 Bukit Timah Road, Singapore 229899, Singapore. Email: wuenlynn.toh@mohh.com.sg

doi: https://doi.org/10.14740/jmc3632 ally in 2011 itself [1]. In 2015 - 2017, the Centre for Disease Control and Prevention estimated the prevalence of primary infertility in women aged 15 - 49 years old was about $19.0 \%$ and the prevalence of secondary infertility about $6.5 \%$ for the same age group [2]. The uptake of ART is set to increase with delayed child-bearing in developing and developed world, and improved accessibility to the technology worldwide. There is also better understanding of ART and thus acceptability of the reproductive methodology.

Current studies state that the incidence of complications from transvaginal oocyte retrieval is low. It is now standard practice to use transvaginal ultrasound to facilitate OR. Follicles are punctured under direct ultrasound guidance to aspirate follicular fluid containing oocyte. This is normally completed under some form of analgesia and anesthesia [3].

Surgical complications arising from OR has indicated that rates of complications are $0.5-8.6 \%$ for vaginal bleeding, 0.02 $1 \%$ for intra-abdominal bleeding, $0.01-0.6 \%$ for pelvic infection and $0.08-0.13 \%$ for ovarian torsion [4-11]. Complications resulting in injuries to the bowels, urinary tract and pelvic vessels are rarer, with only case studies being reported in literature [12-20]. In one case study, they reported a patient with considerable hematuria resulting in cardiovascular compromise and necessitating laparotomy, as a complication of oocyte retrieval [21]. Here, we present a case of bladder injury during oocyte retrieval with a focus on its subsequent management.

\section{Case Report}

\section{Investigations}

A 37-year-old lady was referred to the reproductive medicine clinic for primary infertility. The couple has been trying to conceive for the last 9 years with no success. Infertility workup revealed a normal ovarian reserve (anti-Mullerian hormone (AMH) of $2.6 \mathrm{ng} / \mathrm{mL}$ ) and anovulatory cycles. An ultrasound of her pelvis and hysterosalpingogram showed the presence of a sessile endometrial polyp, a right unicornuate uterus with patent right fallopian tube and a rudimentary noncommunicating left uterine horn (Fig. 1). She was overweight with a body mass index of $32.9 \mathrm{~kg} / \mathrm{m}^{2}$. All other investigations were normal. Her husband's semen analysis was reported to be teratospermia. The patient underwent a hysteroscopic polypectomy to remove the benign polyp prior to commencement of her IVF treatment. 


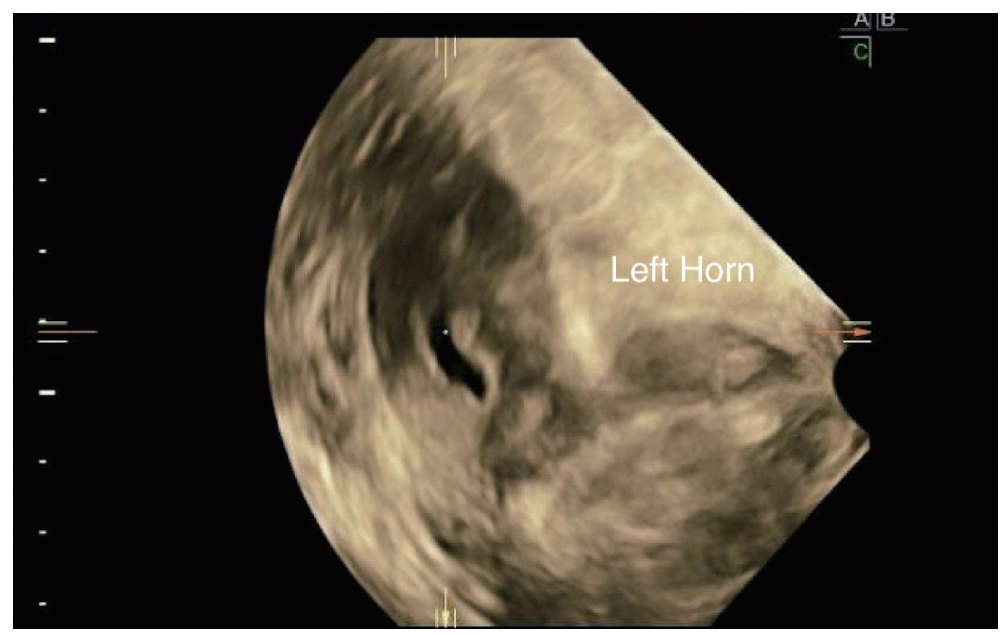

Figure 1. Pelvic ultrasound showing a transverse view of a unicornuate uterus with non-communication left rudimentary horn.

The patient underwent a standard gonadotropin hormonereleasing hormone $(\mathrm{GnRH})$ antagonist protocol, where controlled ovarian stimulation (COS) was achieved with $300 \mathrm{IU}$ of highly-purified human menopausal gonadotrophin (hMG, Menopur, Ferrings, Germany) daily over 9 days, with GnRH antagonist ganirelix $250 \mathrm{mg}$ (Orgalutran, MSD Pharma, Singapore) per day instituted on day 5 of COS. Final oocyte maturation was achieved with $250 \mu \mathrm{g}$ of choriogonadotropin alfa (Ovidrel, Merck Serono, Italy) $36 \mathrm{~h}$ before OR.

Transvaginal OR was performed under intravenous sedation with a $17 \mathrm{G}$ single lumen needle. A total of 14 oocytes were retrieved, 11 of which were mature and inseminated through intracytoplasmic sperm injection, with three blastocysts frozen down for future embryo transfer in view of complications arising from her OR.

\section{Diagnosis and treatment}

During the OR, an inadvertent puncture of the bladder was noted while aspirating the final follicle which was anteriormedially located. Active bleeding from the bladder wall into the bladder cavity was noted on transvaginal ultrasound. A three-way urinary catheter was immediately inserted and bladder washout performed with $1.5 \mathrm{~L}$ of normal saline, with blood clots being evacuated. The patient was then placed on continuous bladder irrigation, administered broad-spectrum antibiotic coverage and admitted for monitoring.

The patient remained hemodynamically stable throughout her admission, with hemoglobin of $11.1 \mathrm{~g} / \mathrm{dL}$ and normal renal function tests. She underwent multiple irrigation of bladder with normal saline over the next 2 days, but was unable to empty her bladder completely independently when trialled without a urinary catheter.

A urological consult was made on day 3 post OR whereupon a computerised tomography cystogram was performed which showed an intact bladder with a $21.8 \mathrm{~mm}$ filling defect within the urinary bladder which is likely representative of a hematoma (Fig. 2). A flexible cystoscopy was performed on day 4 post OR which was largely normal with small patches of posterior wall cystitis (Fig. 3). Post-operatively, the patient managed to have a successful removal of her urinary catheter, with normal voiding function and resolution of any macroscopic hematuria. She was discharged to outpatient care subsequently.

\section{Follow-up and outcome}

Upon the patient's discharge, she was followed up 1 week later where she remained well with mild dysuria which was resolving. No new issues were raised during the consult. She was planned to go through with frozen embryo transfer in 2 months' time and advised to continue with letrozole for ovulation induction in the meantime. However, she did not return for further follow-up and has chosen to postpone further fertility treatment at the time of writing.

\section{Discussion}

Urinary tract injuries post oocyte retrieval are rare and seldom documented. Its incidences may be under-reported as most bladder perforations or injuries may not have any obvious manifestations. However, when an injury results in massive hematuria and hemodynamic instability as reported by Jayakrishnan et al, management needs to be prompt, aggressive and multi-disciplinary [20]. In that case, the patient presented with frank hematuria only 7 days after the oocyte retrieval procedure and required resuscitation and an urgent cystoscopy. The cystoscopy revealed the cause of bleeding to be a pseudoaneurysm which promptly resolved with cystoscopic cauterization. While the exact cause of the pseudoaneurysm was unclear, the authors postulated that the bladder arterial system might have been lacerated during the oocyte retrieval resulting in an arterial leaking into a lower pressured tissue space causing the pseudoaneurysm, similar to how pseudoaneurysms occur during percutaneous nephrolithotomies [20]. 


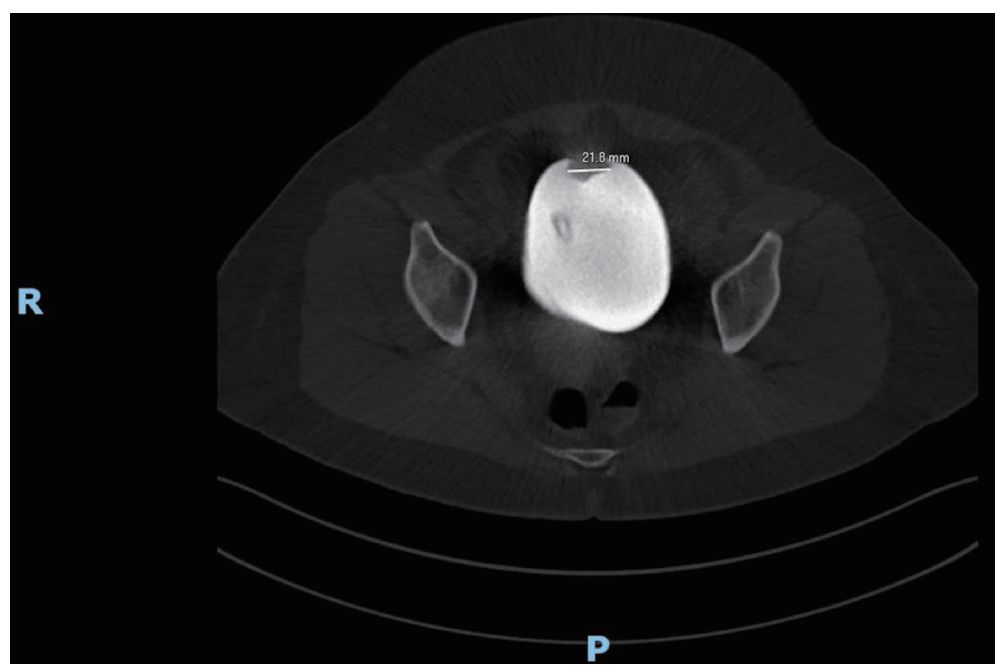

Figure 2. Image showing a $21.8 \mathrm{~mm}$ filling defect within the urinary bladder lumen which likely represents a hematoma.

In our patient, her presentation for the complication was immediate and the bleeding into the bladder cavity was visualized real time on the transvaginal ultrasound. The clinician was quick to identify the issue and started treatment even before the patient presented with hematuria. In this case, an inadvertent bladder-vascular puncture likely led to bleeding into the cavity itself. Therefore, the presentation of manifestations was immediate rather than delayed as per some of the previous case reports [20]. Immediate catheterization and bladder irrigation were important management steps to prevent the accumulation of clots which could potentially block the bladder outlet.

Urology opinion was sought in our patient on post procedure day 3 as her hematuria persisted and she was unable to successfully void independently when her urinary catheter was removed. A multi-disciplinary approach is important in this case to guide management and a computed tomography (CT) urogram was promptly done. While in our patient's case there was no perforation, fistula, aneurysm or bladder mass found; these findings would have vastly altered management. Cystoscopy confirmed the normal findings. Eventually the hematuria resolved with a few days of bladder rest and irrigation, albeit with a minor complication of catheter cystitis.

This was an extremely rare case with serious complication which has no validated or published guidance. The key to management must be early identification and action. Admission to the hospital and stabilization of the hemodynamic status must be prompt. An indwelling urinary catheter must be promptly inserted to prevent over-distension of the bladder and for estimation of the extent of hematuria. Should the hematuria be persistent, a urological consult must be sought. Further modal-

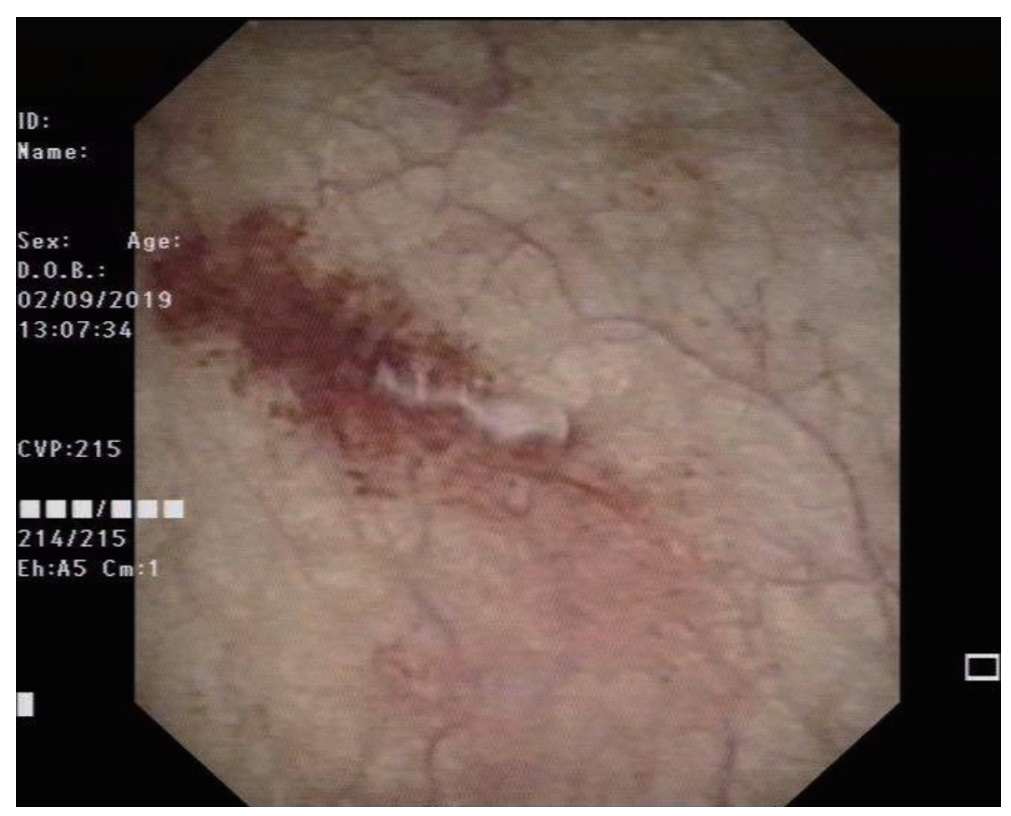

Figure 3. Image showing the cystoscopy finding of erythema over the posterior wall representing posterior wall cystitis. 
ities such as CT urogram and/or cystoscopy may be considered to rule out other causes of persistent hematuria. Furthermore, the cystoscopy may even be a therapeutic modality in cases where a small bleeding source can be seen.

\section{Conclusions}

As with any rare complication, bladder injury during oocyte retrieval must be in a multi-disciplinary team with urology should symptoms persist despite conservative management. Admission for stabilization and insertion of an indwelling urinary catheter must be done as soon as possible. Early identification and promptness to intervention is key. Clinicians need to be mindful of potential injuries to surrounding organs during oocyte retrieval.

\section{Acknowledgments}

The authors would like to acknowledge the valuable input and support from the Reproductive Medicine Department of KK Women's and Children's Hospital, Singapore, Singapore.

\section{Financial Disclosure}

JKYC received salary support from Singapore's Ministry of Health's National Medical Research Council (CSASI-008-2016). The authors have not declared a specific grant for this research from any funding agency in the public, commercial or not-for-profit sectors.

\section{Conflict of Interest}

None to declare.

\section{Informed Consent}

Informed consent has been obtained from the patient for publication.

\section{Author Contributions}

All authors were involved in the intellectual content and production of this case report. WLT collected the relevant data and drafted the manuscript. TXE was involved in editing the manuscript. JKYC was the gynecologist in charge of the patient and was involved in the conception and editing of the manuscript.

\section{Data Availability}

The authors declare that data supporting the findings of this study are available within the article.

\section{References}

1. Adamson GD, de Mouzon J, Chambers GM, ZegersHochschild F, Mansour R, Ishihara O, Banker M, et al. International Committee for Monitoring Assisted Reproductive Technology: world report on assisted reproductive technology, 2011. Fertil Steril. 2018;110(6):1067-1080.

2. NSFG - Listing I - Key Statistics from the National Survey of Family Growth [Internet]. Cdc.gov. 2020 [cited June 28, 2020]. Available from: https://www.cdc.gov/ nchs/nsfg/key_statistics/i_2015-2017.htm\#infertility.

3. Giudice L, Santa E, Pool R. Assessing the medical risks of human oocyte donation for stem cell research. Washington, D.C.: National Academies Press; 2007.

4. Bennett SJ, Waterstone JJ, Cheng WC, Parsons J. Complications of transvaginal ultrasound-directed follicle aspiration: a review of 2670 consecutive procedures. J Assist Reprod Genet. 1993;10(1):72-77.

5. Dicker D, Ashkenazi J, Feldberg D, Levy T, Dekel A, Ben-Rafael Z. Severe abdominal complications after transvaginal ultrasonographically guided retrieval of oocytes for in vitro fertilization and embryo transfer. Fertil Steril. 1993;59(6):1313-1315.

6. Tureck RW, Garcia CR, Blasco L, Mastroianni L, Jr. Perioperative complications arising after transvaginal oocyte retrieval. Obstet Gynecol. 1993;81(4):590-593.

7. Roest J, Mous HV, Zeilmaker GH, Verhoeff A. The incidence of major clinical complications in a Dutch transport IVF programme. Hum Reprod Update. 1996;2(4):345353.

8. Govaerts I, Devreker F, Delbaere A, Revelard P, Englert Y. Short-term medical complications of 1500 oocyte retrievals for in vitro fertilization and embryo transfer. Eur J Obstet Gynecol Reprod Biol. 1998;77(2):239-243.

9. Ludwig AK, Glawatz M, Griesinger G, Diedrich K, Ludwig M. Perioperative and post-operative complications of transvaginal ultrasound-guided oocyte retrieval: prospective study of $>1000$ oocyte retrievals. Hum Reprod. 2006;21(12):3235-3240.

10. Seyhan A, Ata B, Son WY, Dahan MH, Tan SL. Comparison of complication rates and pain scores after transvaginal ultrasound-guided oocyte pickup procedures for in vitro maturation and in vitro fertilization cycles. Fertil Steril. 2014;101(3):705-709.

11. Alvarez C, Sanchez I, Martinez M. Bladder injury after transvaginal oocyte retrieval in IVF cycles. A prospective study. Unidad de Reproduccion Centro Gutenberg.

12. Jones WR, Haines CJ, Matthews CD, Kirby CA. Traumatic ureteric obstruction secondary to oocyte recovery for in vitro fertilization: a case report. J In Vitro Fert Embryo Transf. 1989;6(3):185-187.

13. Van Hoorde GJ, Verhoeff A, Zeilmaker GH. Perforated appendicitis following transvaginal oocyte retrieval for in-vitro fertilization and embryo transfer. Hum Reprod. 1992;7(6):850-851.

14. Akman MA, Katz E, Damewood MD, Ramzy AI, Garcia JE. Perforated appendicitis and ectopic pregnancy following in-vitro fertilization. Hum Reprod. 1995;10(12):3325- 
3326.

15. Coroleu B, Lopez Mourelle F, Hereter L, Veiga A, Calderon G, Martinez F, Carreras O, et al. Ureteral lesion secondary to vaginal ultrasound follicular puncture for oocyte recovery in in-vitro fertilization. Hum Reprod. 1997;12(5):948-950.

16. Miller PB, Price T, Nichols JE, Jr., Hill L. Acute ureteral obstruction following transvaginal oocyte retrieval for IVF. Hum Reprod. 2002;17(1):137-138.

17. Fiori O, Cornet D, Darai E, Antoine JM, Bazot M. Uroretroperitoneum after ultrasound-guided transvaginal follicle puncture in an oocyte donor: a case report. Hum Reprod. 2006;21(11):2969-2971.

18. von Eye Corleta H, Moretto M, D'Avila AM, Berger M.
Immediate ureterovaginal fistula secondary to oocyte retrieval - a case report. Fertil Steril. 2008;90(5):2006 e2001-2003.

19. Fugita OE, Kavoussi L. Laparoscopic ureteral reimplantation for ureteral lesion secondary to transvaginal ultrasonography for oocyte retrieval. Urology. 2001;58(2):281.

20. Jayakrishnan K, Raman VK, Vijayalakshmi VK, Baheti $\mathrm{S}$, Nambiar D. Massive hematuria with hemodynamic instability - complication of oocyte retrieval. Fertil Steril. 2011;96(1):e22-24.

21. Bodri D, Guillen JJ, Polo A, Trullenque M, Esteve C, Coll O. Complications related to ovarian stimulation and oocyte retrieval in 4052 oocyte donor cycles. Reprod Biomed Online. 2008;17(2):237-243. 